\title{
Materials science and engineering graduate core courses in the United States
}

\author{
By Parag Banerjee and Robert M. Briber
}

$\mathrm{T}$ he academic bedrock of a materials science and engineering (MS\&E) $\mathrm{PhD}$ program is a curriculum consisting of a set of core courses. Graduate students are required to take these core courses, followed by electives - courses that are closely related to their topic of research. The rationale for following a core + elective courses structure in a graduate program comes from the early years of MS\&E as it emerged from metallurgy dealing only with metals to an interdisciplinary program with equal footing in metals, ceramics, and polymers. Educators stressed the need to focus on common, fundamental concepts that thread various materials together, rather than to study materials as siloed systems. ${ }^{1}$ Thus, courses dealing with fundamental structure-property relationships in materials, such as solid-state physics, defects, thermodynamics, and kinetics, emerged as core courses. Students could gain an in-depth understanding of the interplay of structure and properties across a range of materials systems.

The goal of this article is to provide a current "state of the core courses" in MS\&E graduate programs in the United States. We note that surveys of top European universities were also conducted. It was found that in European universities where curriculum information was posted online, graduate students were not required to take a set of core courses. In universities in Asia, core courses are an integral part of the MS\&E graduate curriculum, but these vary in number, content, and style from university to university.

Our survey is based on information from $28 \mathrm{MS} \&$ E graduate program websites at US universities. According to the University Materials Council membership roster, ${ }^{2}$ there are currently 113 MS\&E departments in the United States, not all of which may have a graduate program. Another caveat to note is that the

trends described and

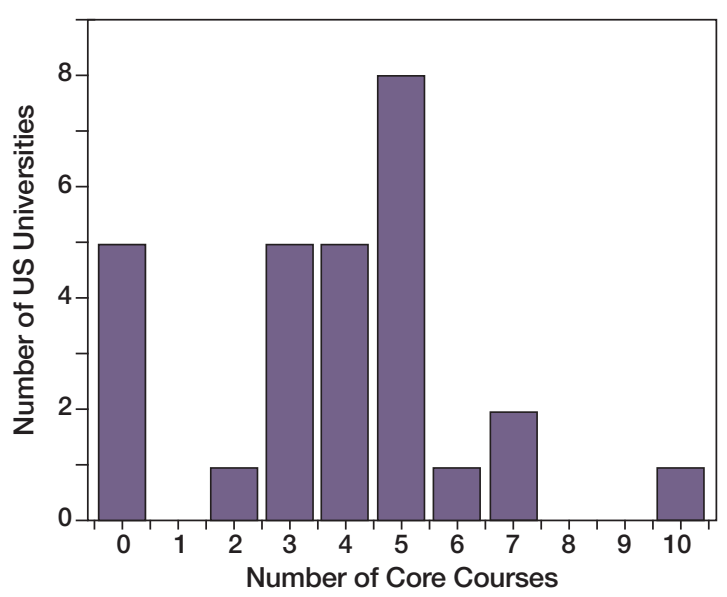

Figure 1. Histogram of the number of core courses offered by US universities in Materials Science and Engineering (MS\&E) graduate programs. Data were obtained from the MS\&E departmental web pages of 28 US universities in 2016. conclusions provided herein are only as good as the quality of data provided on these departmental web pages.

The primary conclusion of this study is that, on average, a typical MS\&E program at a US university consists of five core courses. These courses are spread across three broad categories: (1) structure-property-characterization of materials,
(2) thermodynamics and kinetics, and (3) emerging areas in MS\&E. There is wide variability in the interpretation and breadth of knowledge covered in these courses. This format of core courses is dated and is at least two decades old. It is perhaps time to engage in a national dialogue discussing the purpose and design of a core curriculum in MS\&E, specifically in light of our improved understanding of emerging materials phenomena at multiple length and time scales.

\section{Number of core courses}

The number of core courses offered at the programs examined is plotted as a histogram in Figure 1. The data have a broad spread, with at least five universities requiring no core courses (“0”). On the other hand, at least one university has 10 core courses listed on its website. However, the majority of the 28 US universities explored have between three and five core courses. Considering that a graduate student undertakes coursework in his/her first year, one can conclude that, on average, core courses usually take up the first year of a graduate student's academic load.

It will be instructive to compare this plot with similar analyses in other engineering fields, such as electrical, mechanical, or chemical engineering, which may have multiple subsets of core courses depending on the research area within each of these disciplines. Additionally, as industry demands that materials engineering graduates be specialized in subdisciplines (e.g., soft versus hard matter), academicians should revisit the choice and offerings of core courses and the impact on academic training (depth versus breadth of knowledge), impact on research, and time to graduate. 


\section{Type of core courses}

The core courses fell into three broad categories (Figure 2): (1) structure - property - characterization, (2) thermodynamics and kinetics, and (3) emerging areas.

\section{Structure-property-characterization} This is the largest category of courses deemed to be "core." That such a category is found in core courses should not be surprising, given the taxonomy used in defining MS\&E as a discipline. ${ }^{3}$ As shown in Figure 2, this category can be further divided into subcategories of structure, property, and characterization.

Structure: Traditional courses such as solid-state physics, materials chemistry, and defects in solids fall under the scope of Structure. The syllabus lists the study of atomic arrangement and bonding in solids as the key objective of these courses. Interestingly, at least three universities offer quantum mechanics as a core course. This is not surprising, since MS\&E faculty are diverse in their educational backgrounds, and many have degrees in physics, chemistry, and other fields of engineering. ${ }^{4}$ Furthermore, since current materials research has a significant component dedicated to nanomaterials, subject matter taught in core courses should align with and support this topic as well.

Property: Courses such as mechanical behavior of materials and electronic, optical, and magnetic properties of solids belong to this subcategory. The syllabus typically lists the study of specific properties across the entire spectrum of materials (metals, ceramics, and polymers) as the key objective of these courses. Not surprisingly, research performed by the faculty in these departments influenced many of the courses offered in this subcategory.

Characterization: Courses such as electron microscopy and diffraction, imaging, and structure are categorized as Characterization. The syllabus lists the study of techniques used for characterization of engineering materials as the key objective. Most characterization techniques centered on electron microscopy and x-ray diffraction techniques.

The survey found a lack of inclusion of topics on other spectroscopic and surface

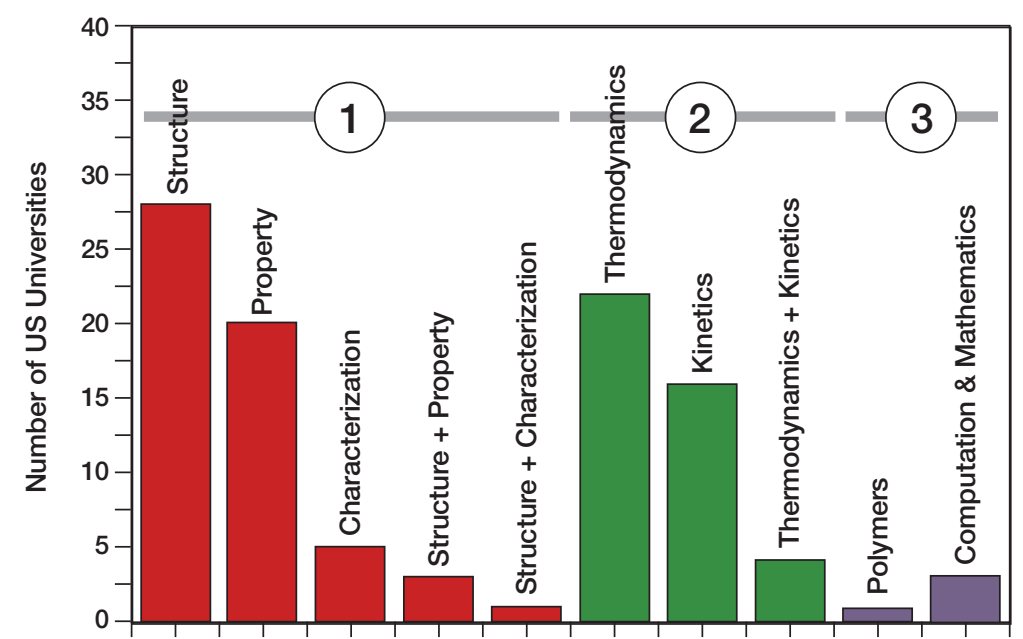

Figure 2. The distribution of core courses in Materials Science and Engineering (MS\&E) $\mathrm{PhD}$ programs at US universities. The three principal categories are (1) structure-propertycharacterization, (2) thermodynamics and kinetics, and (3) emerging areas. Data were obtained from the MS\&E departmental web pages of 28 US universities in 2016.

characterization techniques. As characterization of complex materials becomes increasingly specialized, the suite of techniques should be correspondingly broadened. Characterization courses should include topics in x-ray/ultraviolet photoelectron spectroscopy, optical and mass spectroscopy, atomic force microscopy, and related measurement techniques.

Besides these three subcategories, the survey also found that educators combine topics in structure with properties or characterization. For example, a course deals with crystal structure, bonding, and disorder in solids followed by the implication of structure on various properties (structure + properties). Similarly, a course in crystallography deals extensively with atomic bonding and symmetry principles first, and the latter part of the course is dedicated to x-ray diffraction (i.e., structure + characterization). Finally, we note that the current survey found no core courses dedicated to the synthesis, processing, and manufacturing of materials.

\section{Thermodynamics and kinetics}

The second category is centered around thermodynamics and kinetics. The following topics are commonly listed across all thermodynamics courses: (1) fundamental laws of thermodynamics, (2) equilibrium of solutions, and (3) phase diagrams. In most instances, statistical and molecular thermodynamics form a key component of the syllabus as well. In some instances, thermodynamics of surfaces, heterogeneous reactions, and electrochemistry are taught. For kinetics, the syllabus lists solid-state diffusion as a primary topic of study, followed by theories of nucleation and growth and phase transformation. Finally, in at least two US universities, topics in thermodynamics and kinetics are integrated as a comprehensive course in phase transformation in materials.

\section{Emerging areas}

This was the smallest of the categories surveyed. In at least four instances, we found core courses offered in polymers, and computation and mathematics. We list these courses as emerging areas because research on soft matter and computational materials science is on an uptick. Graduates in MS\&E are expected to have a working knowledge of computational topics, such as multiscale and multiphysics modeling, Monte Carlo techniques, and density functional theory. It is expected that courses in this category (their type and foci) will increase in the coming decades. Exactly how the emerging areas will interrelate with the more traditional categories of structure, properties, characterization, thermodynamics, and kinetics requires a broader dialogue within the MS\&E academic community. 


\section{Summary and final thoughts}

While the overall course objectives are well defined under course descriptions, subtle details, including syllabi and themes, depend on individual faculty, their backgrounds, and research expertise.

An exception to this rule is in the surprising lack of diversity observed in the content of characterization courses. While the bulk of the characterization techniques deal with electron microscopy and $\mathrm{x}$-ray diffraction, the scope of these courses is not keeping pace with advancements in optical spectroscopy and surface characterization techniques.

It is also evident that emerging topics, including soft matter and computational materials science, are now important. These courses need to be effectively integrated into MS\&E core curricula. However, the demands on teaching core courses require a compromise between depth and breadth in the graduate MS\&E curriculum, and this poses a challenge to course restructuring. One approach to optimize and balance these requirements is to rethink courses in thermodynamics, kinetics, characterization, and properties as a function of structure of materials at various length scales.

Such restructuring of the MS\&E core curriculum is outlined as a possibility in
Figure 3, which shows a matrix approach to curricula design. This approach has the advantage of providing a holistic education to graduate students across many materials systems. While the content of core courses would not change, the curriculum could be restructured into modular components. Lecture modules could be organized by assigning length scales to the phenomenon under study. A student would pick a length scale of interest and run through the entire gamut of courses ( $y$-axis in Figure 3) pertaining to it. From an instructor's perspective, the length scale of choice ( $x$-axis in Figure 3) for a lesson module would be the one at which the subject matter could be either observed, measured, or modeled. For instance, in thermodynamics, concepts at the nanoscale would entail teaching statistical mechanics. At the microscale, solution thermodynamics and phase diagrams could be taught spanning both soft and hard matter. Similarly, in kinetics, nanoscale phenomenon would capture order-disorder transformations, whereas microscale kinetics would study Fickian diffusion-driven solidification in metals and alloys.

Structure and modeling courses (double arrow in Figure 3) would adopt this approach as well. For modeling, $a b$ initio techniques would fall under the

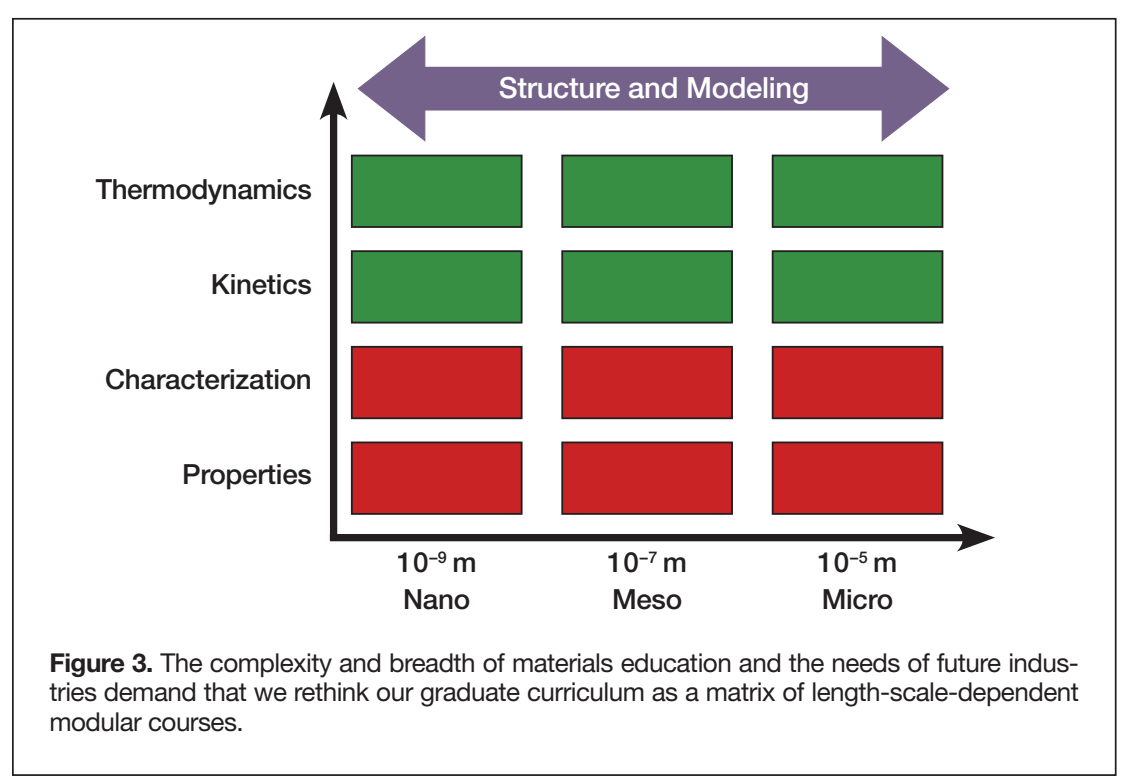

domain of nanoscale modeling, whereas multiphysics and finite-difference/element modeling would fall under the domain of microscale modeling. As is the practice for almost three decades now, ${ }^{3}$ graduate students would continue to develop expertise in the four areas of the materials tetrahedron (structure, properties, synthesis, and performance). Additionally, with the approach described herein, their training would be materials-independent and their expertise would be at multiple length scales relevant to their fields of research.

As materials-based industries become increasingly complex, the demand to innovate complex new materials will continue to rise. ${ }^{5}$ Soft and hard matter and a combination of both will continue to provide solutions to the rising demands of materials functionality and manufacturability and ensure sustained reliability of engineered products. Therefore, training future materials scientists and engineers to innovate across various materials systems is imperative. A length-scale-dependent graduate materials curriculum is an avenue to impart this training. It is the hope of this report that by analyzing the state of core MS\&E graduate courses, a broader dialogue is stimulated in the materials academic community to invent the materials curricula of the future.

\section{Acknowledgments}

Andreea Stoica and Lei Shi, students in the Department of Mechanical Engineering and Materials Science at Washington University in St. Louis, Mo., collected data through analyses of web pages of universities.

\section{References}

1. P.J. Stiles, MRS Bull. 17 (9), 42 (1992).

2. University Materials Council "Education" page (2018), https://www.umatcon.org/files/education.shtml.

3. National Research Council, Materials Science and Engineering: Forging Stronger Links to Users (National Academies Press, Washington, DC, 1999).

4. P. Banerjee, R.M. Briber, MRS Bull. 37 (6), 541 (2012).

5. National Research Council, Materials Science and Engineering for the 1990s: Maintaining Competitiveness in the Age of Materials (National Academies Press, Washington, DC, 1989). 